\title{
Long-Range Ordered Structures of Corannulene Governed by Electrostatic Repulsion and Surface-State Mediation
}

Xiaojie Wen, Yuxuan Lin, Zhichao Huang, Mengxiao Diao, Wenhui Zhao, Jingxin

Dai, Lingbo Xing, Hao Zhu, Zhantao Peng, Dan Liu, Kai Wu*

BNLMS, College of Chemistry and Molecular Engineering, Peking University,

Beijing 100871, China
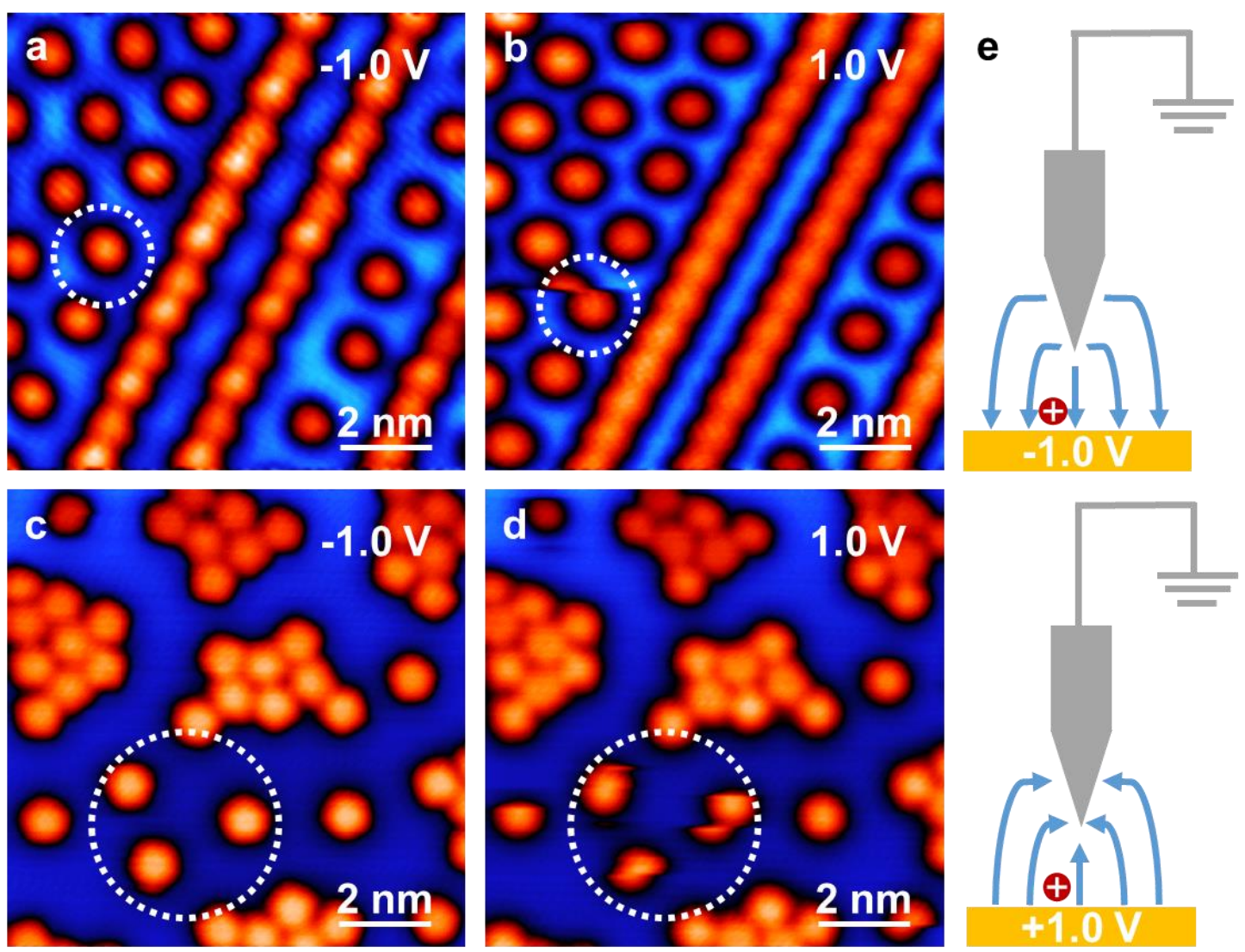

Figure S1. STM images of the CORs on $\mathrm{Cu}(111)$ at (a) negative and (b) positive sample bias. STM images of CORs on $\operatorname{Ag}(111)$ at (c) negative and (d) positive sample bias. $I=$ 30 pA. (e) Schematic illustrations of the positively charged COR under the electric fields established between the tip and substrate at the negative (upper panel) and positive (lower panel) sample bias. 

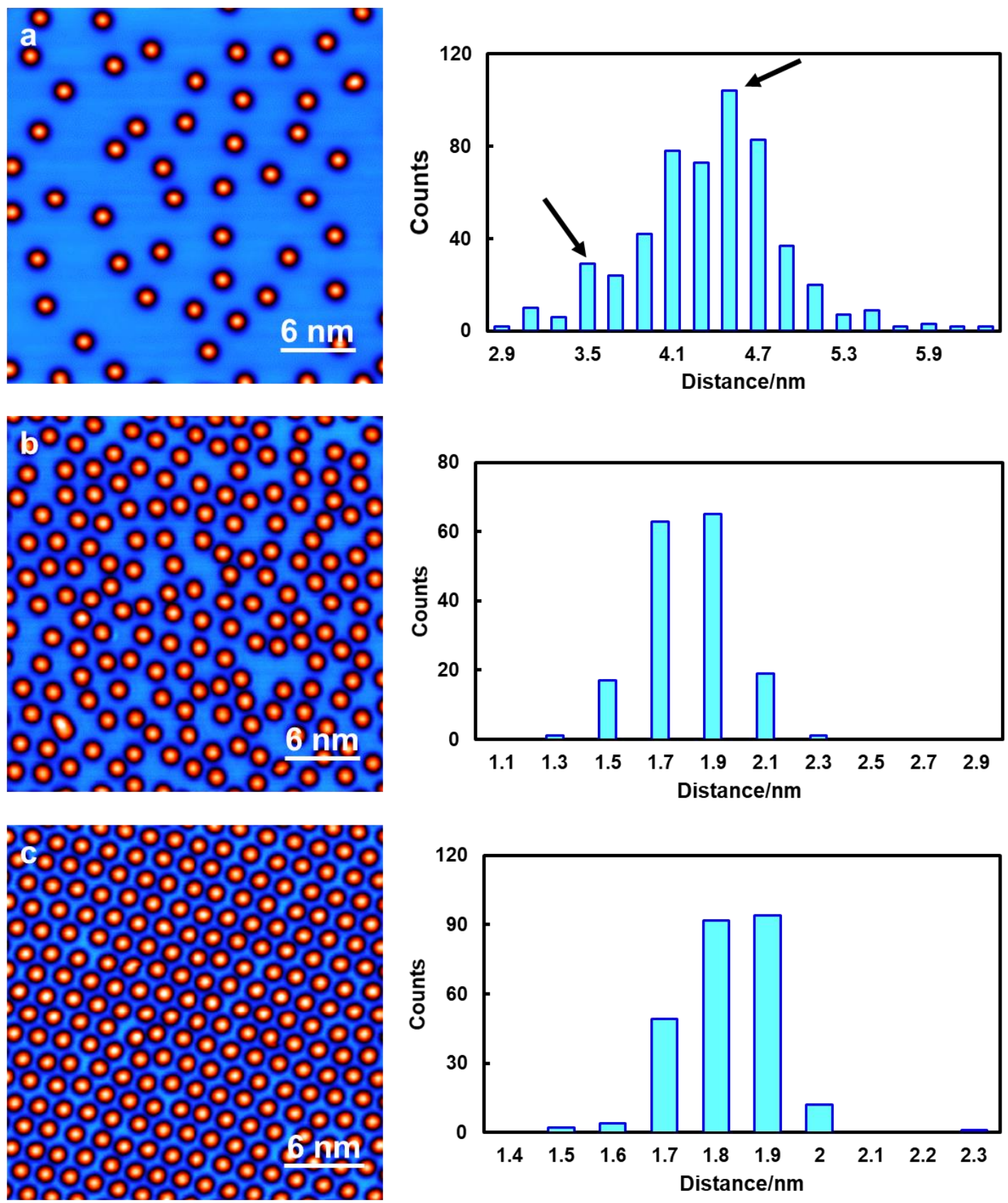

Figure S2. Histograms of the COR nearest-neighbour $(\mathrm{NN})$ separations $\left(f_{\exp }(\mathrm{r})\right)$ and corresponding STM images at 0.05, 0.16 and 0.24 ML. $f_{\exp }(\mathrm{r})$ at 0.05, 0.16 and 0.24 ML are correspondingly achieved by analyses of more than 530, 166 and 254 COR molecules. $V_{\text {bias }}=1.0 \mathrm{~V}, I=30 \mathrm{pA}$. 